\title{
A new curcumin analogue exhibits enhanced antitumor activity in nasopharyngeal carcinoma
}

\author{
YUNBAO PAN $^{1}$, JIAN XIAO ${ }^{2}$, GUANG LIANG ${ }^{2}$, MENGYAO WANG $^{1}$, \\ DUJUAN WANG ${ }^{1}$, SUMEI WANG ${ }^{1}$ and HUILING YANG ${ }^{1}$ \\ ${ }^{1}$ Department of Pathophysiology, Zhongshan School of Medicine, Sun Yat-Sen University, \\ Guangzhou, Guangdong 510080; ${ }^{2}$ School of Pharmacy, Wenzhou Medical College, \\ Wenzhou, Zhejiang 325025, P.R. China
}

Received December 20, 2012; Accepted February 18, 2013

DOI: $10.3892 /$ or.2013.2457

\begin{abstract}
The aim of the present study was to evaluate the antitumor effects of the curcumin analogue GL63 on radioresistant nasopharyngeal carcinoma (NPC) CNE2R cells and parental CNE2 cells. The cell viability and proliferation of NPC cells were detected by MTT assay and colony formation assay. The suppressive effect on tumor growth was examined using in vivo subcutaneously inoculated NPC tumor models using nude mice. The cell cycle distribution was detected using flow cytometry. Apoptosis was examined by Hoechst 33342 and Annexin V/PI staining assay. The protein expression of endoplasmic reticulum (ER) stress pathway markers, XBP-1, ATF-4 and CHOP, were examined by western blotting. A growth inhibitory effect was observed following treatment with GL63 in a dose-dependent manner and was more potent when compared to curcumin. GL63 at $5 \mu \mathrm{M}$ induced significant G2/M arrest and apoptosis in NPC. The tumor-suppressive activity of GL63 in NPC xenograft models was more potent when compared to curcumin. Furthermore, GL63 induced an ER stress response, upregulation of CHOP, XBP-1 and ATF-4 expression, while the same concentration of curcumin had no effect on ER stress. These results suggest that GL63 has more potent antitumor activity than curcumin, which is associated with activation of ER stress, induction of $\mathrm{G} 2 / \mathrm{M}$ arrest and apoptosis in NPC cells.
\end{abstract}

\section{Introduction}

Nasopharyngeal carcinoma (NPC) is the most common malignant tumor in Southeast Asia particularly in South China (1). Chemotherapy and radiotherapy are the main treatment strate-

Correspondence to: Professor Huiling Yang, Department of Pathophysiology, Zhongshan School of Medicine, Sun Yat-Sen University, Zhongshan 2nd Road, Guangzhou, Guangdong 510080, P.R. China

E-mail: hlyangsums@hotmail.com

Key words: nasopharyngeal carcinoma, curcumin, Epstein-Barr virus-associated malignancy, apoptosis, endoplasmic reticulum stress gies for NPC (2); however, radioresistance remains a serious obstacle to successful treatment in the clinic $(2,3)$. To develop better approaches, it is important to seek innovative therapeutics for NPC.

An increasing amount of attention has been paid to the use of complementary and alternative medicine as a part of the treatment strategy for various types of cancers (4). Curcumin, a phenolic compound from the plant Curcuma longa, is a well-known food additive and constituent of traditional medicine, and has been reported to inhibit cell proliferation and induce apoptosis in many types of human cancers $(5,6)$. However, the exact molecular mechanisms involved in the tumor-suppressive effects of curcumin remain to be identified. Although curcumin is remarkably non-toxic and has promising anticancer activities, preclinical and clinical studies indicate that its poor bioavailability and pharmacokinetic profiles due to its instability under physiological conditions have limited its application in antitumor therapies (7). It has been previously reported that curcumin exerts its pro-apoptotic effect by inducing endoplasmic reticulum ER stress in human leukemia HL-60 cells (8). Furthermore, it has been reported that ER stress contributes to radiosensitization (9). Therefore, curcumin analogues with higher potency that specifically activate the ER stress pathway are needed as effective therapeutic agents for NPC.

Recently, more effort has been paid to the chemical modification of curcumin to identify potential analogues with better bioavailability and antitumor activities $(10,11)$. We also designed and synthesized a series of mono-carbonyl analogues of curcumin by deleting the $\beta$-diketone moiety $(12,13)$. Our preliminary studies revealed that several mono-carbonyl analogues not only have enhanced stability and antitumor activities in vitro but also have better pharmacokinetic profiles in vivo. One such compound, (1E,4E)-1,5-bis(2-bromophenyl) penta-1,4-dien-3-one (GL63) (Fig. 1) was synthesized in our laboratory as part of a series of novel curcumin analogues. We demonstrated that GL63, as a new curcumin analogue, was more active than curcumin in the inhibition of cell proliferation and induction of apoptosis in human hepatocellular carcinoma HepG2 cells and human lung epithelial H460 cancer cells $(14,15)$. In the present study, we characterized the biologic activity of GL63 on NPC cells. Our data indicated that 
GL63 inhibits cell viability, proliferation, induces cell cycle arrest and apoptosis. GL63 displayed a greater specificity for activating the ER stress pathway than curcumin. Treatment of NPC with GL63 also resulted in enhanced tumor growth suppression in vivo. Our data suggest that GL63 represents a promising lead compound that can be optimized further for development as a therapeutic agent for NPC.

\section{Materials and methods}

Materials. Cell culture reagents and fetal bovine serum (FBS) were obtained from Invitrogen (Carlsbad, CA, USA). Antibodies against CHOP, XBP-1, ATF-4, lamin B and horseradish peroxidase (HRP)-conjugated donkey anti-goat $\operatorname{IgG}$ were from Santa Cruz Biotechnology (Santa Cruz, CA, USA). Western Lightning Chemiluminescence Plus reagent was from Perkin-Elmer Life Sciences (Boston, MA, USA). Annexin V/PI kit was from BD Biosciences (Palo Alto, CA, USA). Curcumin, dimethyl sulfoxide (DMSO) and 3-(4,5-dimethylthiazol2-yl)-2,5-diphenyltetrazolium bromide (MTT) were from Sigma-Aldrich, Inc. (St. Louis, MO, USA). GL63 was stored in our laboratory as previously described (15).

Cell lines and culture conditions. The NPC cell line, CNE2, was obtained from the Experimental Animal Center of Sun Yat-Sen University. Radioresistant NPC cell line, CNE2R, was established in our laboratory as previously described (16). The NPC cells were cultured in RPMI-1640 medium with $10 \%$ FBS and antibiotics $(100 \mathrm{U} / \mathrm{ml}$ of penicillin and $100 \mu \mathrm{g} / \mathrm{ml}$ of streptomycin) in cell culture incubators that were set at $37^{\circ} \mathrm{C}$ and aired with $5 \% \mathrm{CO}_{2}$.

MTT cell viability assay. The MTT assay was used to evaluate cell viability as previously described (17). Briefly, NPC cells were seeded in 96-well plates (2,000 cells/well) in RPMI-1640 medium with $10 \%$ FBS. The following day the cells were treated with GL63 and curcumin as indicated and incubated for $48 \mathrm{~h}$. MTT $(20 \mu \mathrm{l})(5 \mathrm{mg} / \mathrm{ml})$ was added to each well and incubation was carried out for $3.5 \mathrm{~h}$. The medium was discarded and $150 \mu \mathrm{l}$ of DMSO was added to each well, and incubated for $10 \mathrm{~min}$. The absorbance was read at $570 \mathrm{~nm}$. The half-maximal inhibitory concentration $\left(\mathrm{IC}_{50}\right)$ was used as the concentration of drug required to obtain $50 \%$ of maximal inhibition in cell viability.

Colony formation assay. We performed a colony formation assay as previously described (18). In brief, the NPC cells (200 cells/well) were plated in a 6-well plate for growth analysis in RPMI-1640 medium with 10\% FBS. The following day the cells were treated with $1 \mu \mathrm{M}$ GL63 or $1 \mu \mathrm{M}$ curcumin and incubated for $24 \mathrm{~h}$. The NPC cells were grow at $37^{\circ} \mathrm{C}$ for 14 days. The effect of the drugs on growth was determined by colony growth. Colonies were stained with Giemsa dye and scored by counting with an inverted microscope, using the standard definition that a colony consists of 50 or more cells. Numbers were normalized to the percentage of the colonies formed in DMSO treatment.

Cell cycle analysis by flow cytometry. Following treatment with $5 \mu \mathrm{M}$ of curcumin or GL63, cells were collected and fixed overnight in $75 \%$ cold ethanol at $4^{\circ} \mathrm{C}$. Cells were washed twice in cold PBS and labeled with propidium iodide (PI, Sigma) as previously described (17), and analyzed immediately after staining using Epics Elite flow cytometer (Coulter Diagnostics, Opa Locka, FL, USA) and WinMDI29 software.

Measurement of apoptosis by Hoechst staining and flow cytometry. To detect apoptosis, we performed nuclear staining as previously described (18). Cells exposed to GL63 or curcumin $(5 \mu \mathrm{M})$ for $48 \mathrm{~h}$ were washed twice with PBS and fixed with methanol for $15 \mathrm{~min}$. The fixed cells were then washed again with PBS and stained with $10 \mu \mathrm{g} / \mathrm{ml}$ of Hoechst 33342 for $15 \mathrm{~min}$. The cells were examined under a fluorescence microscope (Olympus, DX50).

Flow cytometric analysis, as previously described (17), was used to differentiate between living, early apoptotic, late apoptotic/necrotic and necrotic cells by staining with Annexin V and PI. Briefly, after treatment with $5 \mu \mathrm{M}$ GL63 or curcumin for $48 \mathrm{~h}$, all of the cells were collected and resuspended in $100 \mu \mathrm{l}$ binding buffer containing Annexin and PI according to the manufacturer's recommendations. Quantification of Annexin V and PI binding was performed by a FACScan.

Western blot analysis. Following treatment with 5, 10 and $20 \mu \mathrm{M}$ curcumin or GL63, the culture medium was collected and cells were washed with ice-cold phosphate-buffered saline (PBS). The whole cell lysate was prepared using lysis buffer containing $150 \mathrm{mM} \mathrm{NaCl}, 1.0 \%$ Nonidet P-40, $0.5 \%$ sodium deoxycholate, $0.1 \%$ sodium dodecyl sulphate (SDS), $50 \mathrm{mM}$ Tris, pH 8.0 and protease and phosphatase inhibitors. Nuclear extracts were isolated as previously described (15). The protein concentration was measured using Bio-Rad protein assay reagent. The nuclear proteins (15 $\mu \mathrm{g}$ of proteins) were resolved on 10\% Bis-Tris Criterion XT gels (Bio-Rad) and transferred onto nitrocellulose membranes. Immunoblots were blocked with 5\% non-fat milk in Tris-buffered saline (TBS) for $1 \mathrm{~h}$ at room temperature, and then incubated with primary antibodies against CHOP, XBP-1, ATF-4 and Lamin B at $4-8^{\circ} \mathrm{C}$ overnight. Immunoreactive bands were detected using HRP-conjugated secondary antibodies with the Western Lightning Chemiluminescence Plus reagent.

Tumorigenicity assays in nude mice. The NPC xenograft model was used to evaluate tumor growth-suppressive activity as previously described (19). Briefly, female 4- to 6-week-old nude mice were obtained from the Animal Center of Sun Yat-Sen University (Guangdong, China) and divided into four experimental groups ( $\mathrm{n}=4$ for each group). NPC cells were divided into the DMSO (control) group, curcumin $(5.0 \mu \mathrm{M})$ group and GL63 $(2.5$ and $5.0 \mu \mathrm{M})$ groups. After treatment for $24 \mathrm{~h}$, cells $\left(2 \times 10^{6}\right)$ were harvested and injected s.c. into the right flank of each mouse. Tumor volumes were measured every 2 days by measuring the length (L) and width (W). The tumor volume was calculated as $\mathrm{LW}^{2} / 2$. At the end of the experiment, the mice were sacrificed, and the tumors were removed for weighing.

Statistical analysis. Results are shown as means \pm standard error (SE). Statistical analysis for the results was carried out using the Student's t-test when only two groups were compared, 
A<smiles>COc1cc(CCC(O)CC(=O)CCc2ccc(O)c(O)c2)ccc1O</smiles><smiles>OC(/C=C/c1ccccc1Br)/C=C/c1ccccc1Br</smiles>
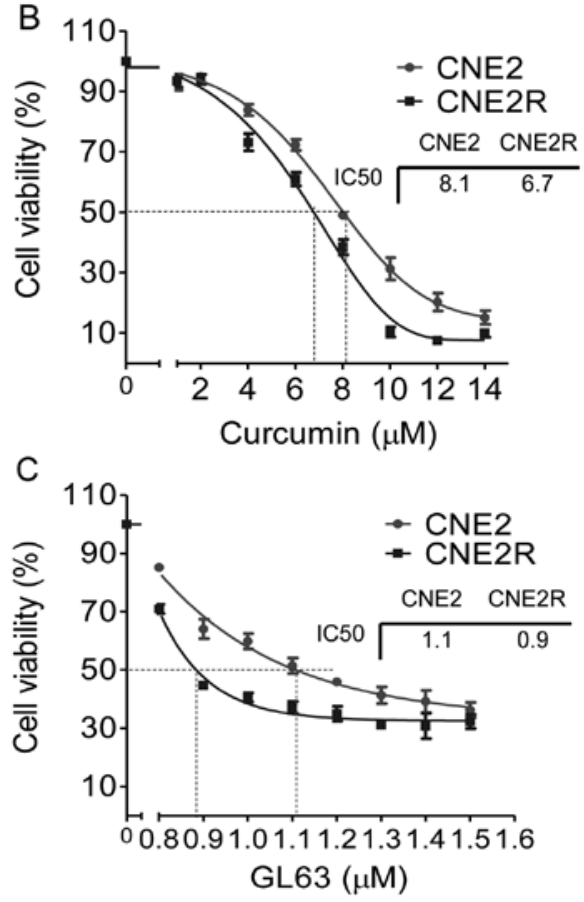

Figure 1. GL63 inhibits NPC cell viability. (A) Schematic presentation of GL63. The effects of (B) curcumin and (C) GL63 on the cell viability of NPC cells were determined by MTT assay. Cell viability was determined from 3 independent experiments performed in triplicate.

or one-way analysis of variance when more than two groups were compared. Differences between groups were stated to be statistically significant at $\mathrm{P}<0.05$.

\section{Results}

GL63 inhibits NPC cell viability and proliferation more potently than curcumin. We examined the growth suppressive activities of curcumin and GL63 (Fig. 1A) in two human NPC cell lines, CNE2 and CNE2R. After treatments for $48 \mathrm{~h}$, both GL63 and curcumin caused inhibition of cell viability in the two NPC cell lines (Fig. 1B and C). However, GL63 exhibited greater inhibition than curcumin. $\mathrm{IC}_{50}$ values for GL63 were $1.1 \mu \mathrm{M}$ in the CNE2 cells and $0.9 \mu \mathrm{M}$ in the CNE2R cells, respectively, which were substantially more potent than curcumin $\left(\mathrm{IC}_{50}\right.$ values 8.1 and $\left.6.7 \mu \mathrm{M}\right)$.

We further performed a colony formation assay to test the effect of GL63 on NPC cell proliferation. The clonogenic survival rate decreased 71 and $100 \%$ following treatment with 0.5 and $1 \mu \mathrm{M}$ GL63, while the survival rate was reduced only 10 and $19 \%$ following treatment with the same concentrations of curcumin (Fig. 2A). We observed similar results for the CNE2R cells; a 93 and $100 \%$ decrease in the survival rate was noted after exposure to 0.5 and $1 \mu \mathrm{M}$ GL63, while only a 7 and $15 \%$ reduction was noted after exposure to the same doses of curcumin. These results demonstrated that GL63 was more potent than curcumin in inhibiting cell viability and proliferation in NPC cells.

GL63 is more potent than curcumin in inducing NPC cell cycle arrest. Cell cycle distribution of CNE2 and CNE2R cells was assessed following a 24-h treatment with $5 \mu \mathrm{M}$ GL63 or curcumin by flow cytometry. Treatment with GL63 resulted in an increase in the percentage of cells in the $\mathrm{G} 2 / \mathrm{M}$ phase from 3.6 to $21.5 \%$ in CNE2 and from 6.1 to $26.6 \%$ in CNE2R cells, whereas $5 \mu \mathrm{M}$ curcumin did not cause a significant change in the cell cycle distribution, indicating that GL63 induces G2/M phase arrest in NPC cells (Fig. 2B). These data indicate that GL63 exerts antitumor activity through cell cycle arrest.

GL63 promotes NPC cell apoptosis more potently than curcumin. We next aimed to determine whether the GL63induced inhibition of cell viability occurs through increased apoptosis. We first analyzed the effect of GL63 on the viability of cells using Hoechst fluorescence. In agreement with our MTT data, GL63 markedly decreased the viable cell number; there was a 68 and $81 \%$ reduction in CNE2 and CNE2R cells, while only a 24 and $27 \%$ reduction was noted following treatment with the same dose of curcumin (Fig. 3A). Furthermore, GL63 induced morphological changes, which were characteristic of apoptosis in the two NPC cell lines (Fig. 3A). In contrast, the curcumin-treated cells displayed excellent growth characteristics.

Flow cytometric analysis of NPC cells exposed to GL63 confirmed the morphological observations noted above. As shown in Fig. 3B, after treatment with $5 \mu \mathrm{M}$ of curcumin for $48 \mathrm{~h}$, the apoptosis rate exhibited no difference when compared with the control group. However, following treatment with the same concentration of GL63, the apoptotic ratios were 47.7\% (CNE2) and 65.3\% (CNE2R), respectively, which confirmed the enhanced activity of induction of apoptosis by GL63 in NPC.

GL63 represses the tumorigenicity of NPC cells more potently than curcumin. We explored the tumor-suppressive activity of the curcumin analogue in NPC xenograft models. CNE2 and 

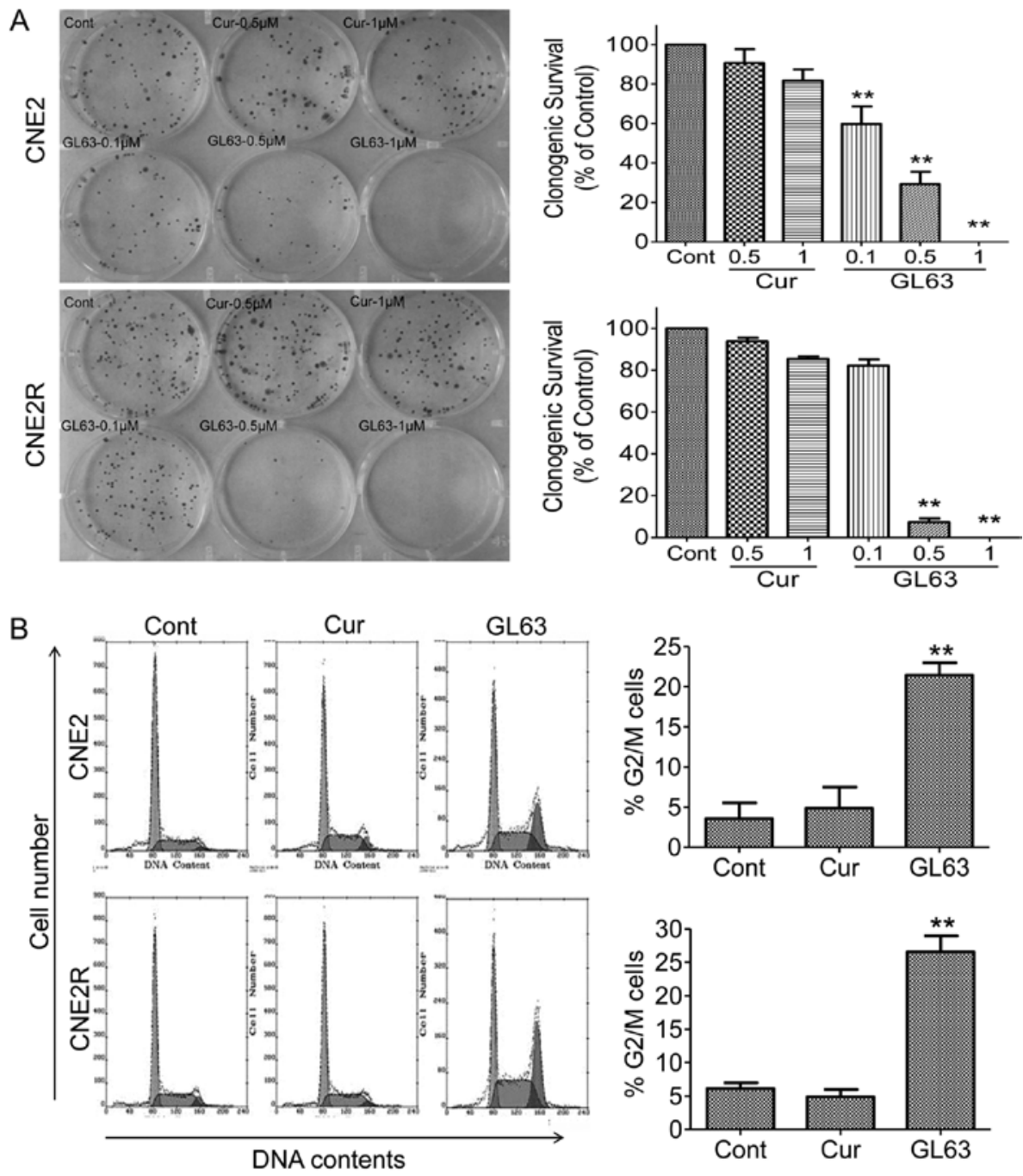

Figure 2. GL63 inhibits NPC cell proliferation. (A) The effects of GL63 on cell proliferation were examined by clone formation assay. Left: NPC cells were treated with DMSO control or the indicated concentrations of GL63 or curcumin (Cur) for $24 \mathrm{~h}$. Ten days later, cells were fixed in methanol and stained with Giemsa dye and scored by counting with an inverted microscope. Right: quantification of the colony staining. (B) The effects of GL63 and curcumin on the cell cycle distribution of NPC cells were determined by flow cytometric analysis. Left: NPC cells were treated with control (DMSO) or $5 \mu$ M of GL63 or curcumin for $24 \mathrm{~h}$, then fixed in ethanol and stained with propidium iodide. Right: quantification of cells in the G2/M phase. All data represent 3 independent experiments; mean $\pm \mathrm{SD}$. ${ }^{* *} \mathrm{P}<0.01$.

CNE2R cells were treated with DMSO control or curcumin $(5 \mu \mathrm{M})$ or GL63 $(2.5$ or $5 \mu \mathrm{M})$ for $24 \mathrm{~h}$. The cells were then implanted into nude mice. Tumor growth was observed in the control and the drug-treated mice. GL63 effectively suppressed the growth of tumors in mice bearing the CNE2 (Fig. 4A) and CNE2R cells (Fig. 4B), reducing the tumor weight by $54.2 \%$ (CNE2) and 70.8\% (CNE2R), respectively, compared with the control group (Fig. 4C). Equal concentrations of curcumin showed only a tumor growth inhibition of $37.6 \%$ in the CNE2 tumors and $8.5 \%$ in the CNE2R tumors, respectively (Fig. 4C). Furthermore, no tumor development was noted in mice of the $5 \mu \mathrm{M}$ GL63 group, suggesting that GL63 is more effective than curcumin in inhibiting NPC tumorigenicity.

Specific activation of the ER stress pathway by GL63. Induction of downstream transcription factors, GRP78, XBP-1, ATF-4 and CHOP, are markers for the activation of ER stress $(20,21)$. We compared the effects of GL63 and curcumin on the ER stress pathway in NPC cells. GL63 significantly increased the levels of CHOP, XBP-1, ATF-4 protein at 10 and $20 \mu \mathrm{M}$ in CNE2 (Fig. 5A) and CNE2R cells (Fig. 5B), whereas curcumin treatment did not cause any change in the expression levels of these proteins, even at $20 \mu \mathrm{M}$ (Fig. 5). These results suggest that GL63-induced ER stress represents a major cellular mechanism of its antitumor activity.

\section{Discussion}

Since curcumin is poorly absorbed, more potent and soluble curcumin analogues have been developed (7). In our previous studies $(14,15)$, a series of curcumin analogues were designed by the deletion of the highly reactive $\beta$-diketone moiety in the structure of curcumin which is considered to be responsible for the in vitro instability and the in vivo pharmacokinetic disadvantages. In the present study, we demonstrated that the novel curcumin analogue GL63 significantly inhibited NPC 
A
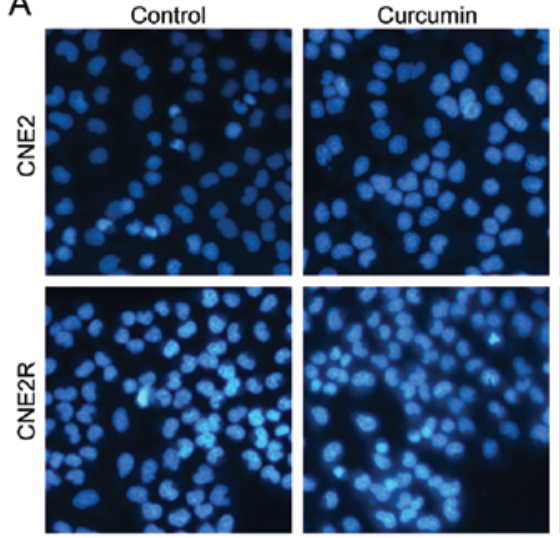

B

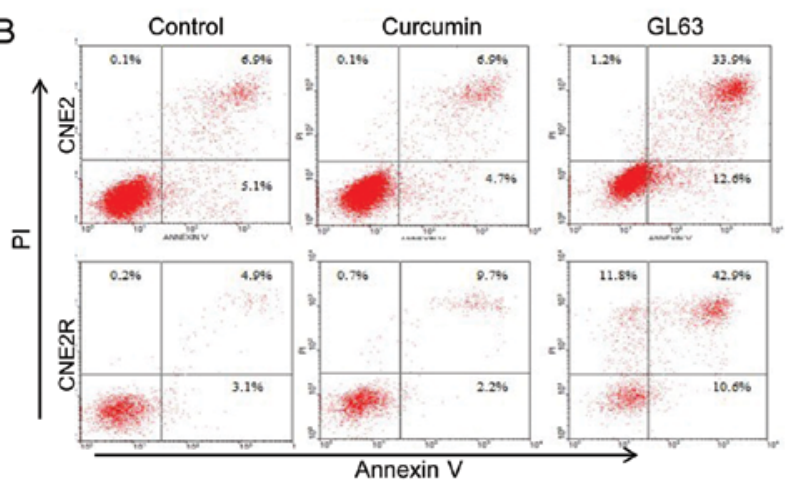

GL63
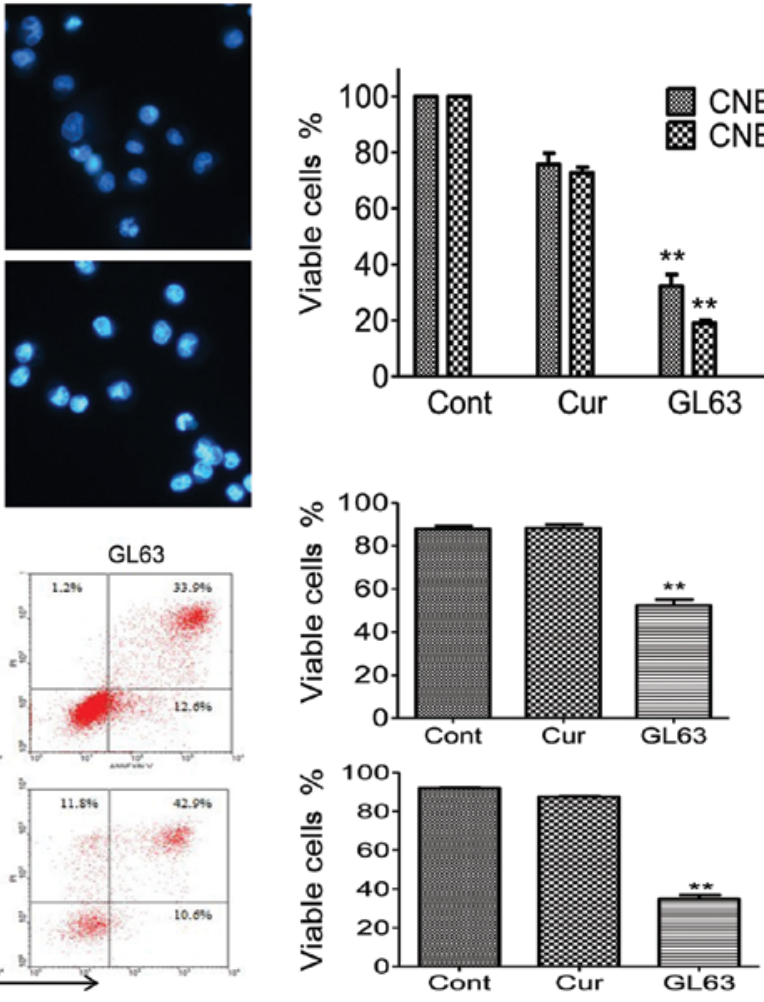

Figure 3. GL63 induces NPC cell apoptosis. NPC cells were treated with DMSO control or $5 \mu$ M of GL63 or curcumin (Cur) for 48 h, then stained with Hoechst 33342 and apoptosis was determined by (A) fluorescence microscopy or (B) staining with Annexin V/PI and detected by flow cytometry. Right: quantification of cell apoptosis. All data represent 3 independent experiments; mean $\pm \mathrm{SD}$. ${ }^{* *} \mathrm{P}<0.01$.

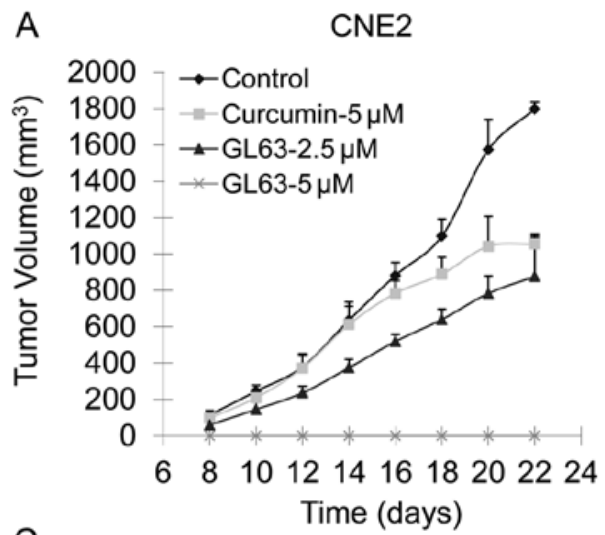

C CNE2

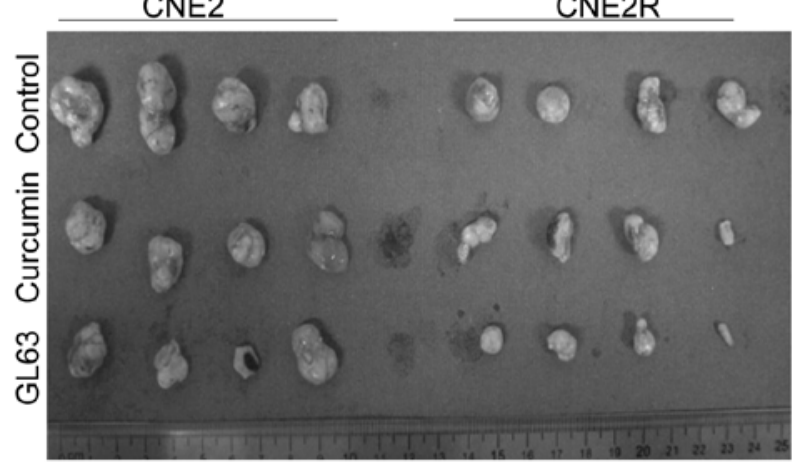

B
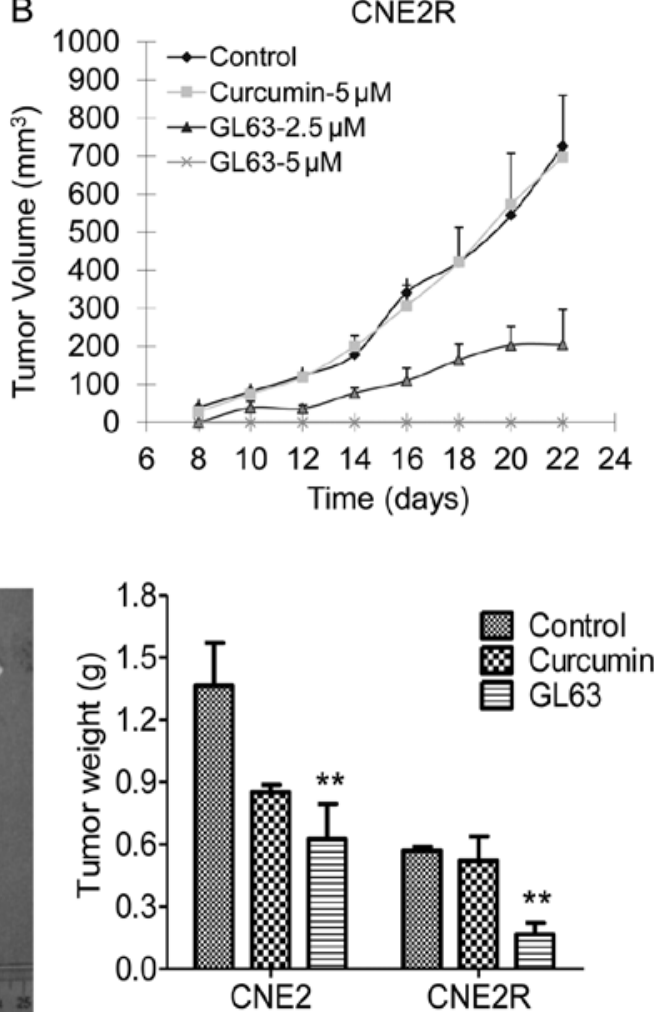

Figure 4. GL63 inhibits the tumorigenicity of NPC cells in nude mice. (A) CNE2 and (B) CNE2R cells were treated with the indicated concentrations of curcumin or GL63 for $24 \mathrm{~h}$. The cells were then harvested and injected s.c. into the right flank of mice. Tumor volumes were monitored for the indicated number of days. (C) Tumor weight was measured at the end of the experiment. Bars, $\mathrm{SE},{ }^{* *} \mathrm{P}<0.01$. 
A

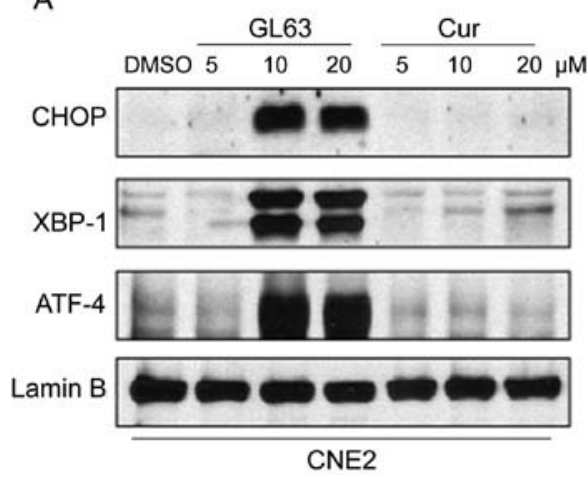

B

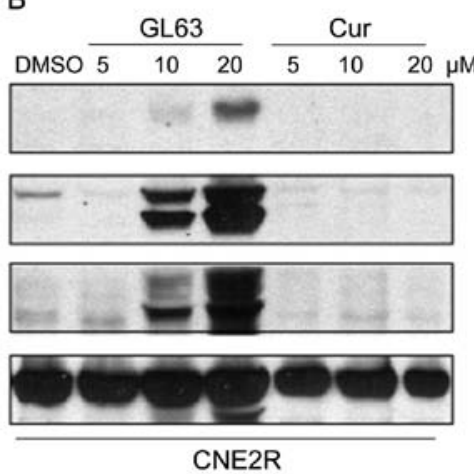

Figure 5. GL63 activates the ER stress pathway in NPC cells. (A) CNE2 and (B) CNE2R cells were treated with the indicated doses of GL63 or curcumin (Cur) for $24 \mathrm{~h}$, and, CHOP, XPB-1, ATF-4 levels were determined by western blot analysis. Lamin B was used as a protein loading control.

cell viability, proliferation, and induced cell cycle arrest, while curcumin at the same dose did not have the same therapeutic effects.

Further study confirmed that GL63 was directly involved in inhibiting tumor growth in vivo. To the best of our knowledge, this is the first study to demonstrate that a curcumin analogue has the capability for enhanced antitumor activity in radioresistant NPC cells.

In addition to GL63, other curcumin analogues have been reported $(22,23)$. However, none of the current curcumin analogues have been reported to be able to induce cell cycle arrest and apoptosis in NPC. Our results revealed that GL63 induces $\mathrm{G} 2 / \mathrm{M}$ phase arrest and apoptosis in CNE2 and CNE2R cells. Mammalian cells exhibit significant variation in radiosensitivity as the cell cycle progresses. Cells in the G2/M phase are the most radiosensitive (24). These findings show that accumulation of cells in the most radiosensitive $\mathrm{G} 2 / \mathrm{M}$ phase caused by GL63 may enhance the radiosensitization effect in NPC cells.

A recent study found that intracellular organelles, including the ER, promote cell apoptosis signals. The unfolded protein response (UPR) is an intracellular signaling pathway, which regulates the accumulation of unfolded or misfolded proteins in the ER and plays an important role in regulating cell growth, differentiation and apoptosis $(25,26)$. Therefore, the possibility that GL63 induces apoptosis via ER stress was examined. CHOP is a typical ER stress-regulated protein involved in ER stress-induced apoptosis (27). Our results concerning CHOP induction by GL63 suggest that GL63 may trigger ER stress. GL63 also increased the levels of XBP-1 and ATF-4, both of which are proteins increased during ER stress. However, curcumin at the same concentration had no effect on these ER stress markers, suggesting that the enhanced antitumor effect of GL63 involves ER stress. Furthermore, ER stress signaling appears to play an important role in proliferation, chemoresistance, and radioresistance in cancer cells $(28,29)$. We found that GL63 not only affected the CNE2 cells, but also had a markedly suppressive effect on radioresistant CNE2R cells, which may have resulted from the induction of the ER stress pathway.

Taken together, our results indicate that GL63 exhibits enhanced antitumor activity in NPC cells. Our findings demonstrated that GL63 activates ER stress and supports
GL63 as an anticancer agent with important clinical relevance. The administration of GL63 is a potential therapeutic regimen for NPC.

\section{Acknowledgements}

The present study was supported by the National Natural Science Foundation of China (81071837 and 30670627); the Natural Science Foundation of Guangdong Province, China (9251008901000005 and 06021210) and the Scientific and Technological Project of Guangdong, China (2008A030201009 and 2010B050700016) (H.Y.).

\section{References}

1. Lo KW, Chung GT and To KF: Deciphering the molecular genetic basis of NPC through molecular, cytogenetic, and epigenetic approaches. Semin Cancer Biol 22: 79-86, 2012.

2. Hui EP, Ma BB, Leung SF, et al: Randomized phase II trial of concurrent cisplatin-radiotherapy with or without neoadjuvant docetaxel and cisplatin in advanced nasopharyngeal carcinoma. J Clin Oncol 27: 242-249, 2009.

3. Yip KW, Mocanu JD, Au PY, et al: Combination Bcl-2 antisense and radiation therapy for nasopharyngeal cancer. Clin Cancer Res 11: 8131-8144, 2005.

4. Lev-Ari S, Lichtenberg D and Arber N: Compositions for treatment of cancer and inflammation. Recent Pat Anticancer Drug Discov 3: 55-62, 2008.

5. Gandhy SU, Kim K, Larsen L, Rosengren RJ and Safe S: Curcumin and synthetic analogs induce reactive oxygen species and decrease specificity protein $(\mathrm{Sp})$ transcription factors by targeting microRNAs. BMC Cancer 12: 564, 2012.

6. Binion DG, Otterson MF and Rafiee P: Curcumin inhibits VEGF-mediated angiogenesis in human intestinal microvascular endothelial cells through COX-2 and MAPK inhibition. Gut 57: 1509-1517, 2008.

7. Anand P, Kunnumakkara AB, Newman RA and Aggarwal BB: Bioavailability of curcumin: problems and promises. Mol Pharm 4: 807-818, 2007.

8. Pae HO, Jeong SO, Jeong GS, et al: Curcumin induces proapoptotic endoplasmic reticulum stress in human leukemia HL-60 cells. Biochem Biophys Res Commun 353: 1040-1045, 2007.

9. Isohashi F, Endo H, Mukai M, Inoue T and Inoue M: Insulin-like growth factor stimulation increases radiosensitivity of a pancreatic cancer cell line through endoplasmic reticulum stress under hypoxic conditions. Cancer Sci 99: 2395-2401, 2008.

10. Yogosawa S, Yamada Y, Yasuda S, Sun Q, Takizawa K and Sakai T: Dehydrozingerone, a structural analogue of curcumin, induces cell-cycle arrest at the G2/M phase and accumulates intracellular ROS in HT-29 human colon cancer cells. J Nat Prod 75: 2088-2093, 2012. 
11. Subramaniam D, May R, Sureban SM, et al: Diphenyl difluoroketone: a curcumin derivative with potent in vivo anticancer activity. Cancer Res 68: 1962-1969, 2008.

12. Liang G, Yang S, Zhou H, et al: Synthesis, crystal structure and anti-inflammatory properties of curcumin analogues. Eur J Med Chem 44: 915-919, 2009.

13. Liang G, Shao L, Wang Y, et al: Exploration and synthesis of curcumin analogues with improved structural stability both in vitro and in vivo as cytotoxic agents. Bioorg Med Chem 17: 2623-2631, 2009.

14. Xiao J, Chu Y, Hu K, et al: Synthesis and biological analysis of a new curcumin analogue for enhanced anti-tumor activity in HepG 2 cells. Oncol Rep 23: 1435-1441, 2010.

15. Xiao J, Tan Y, Pan Y, et al: A new cyclooxygenase-2 inhibitor (1E,4E)-1,5-bis(2-bromophenyl)penta-1,4-dien-3-one (GL63) suppresses cyclooxygenase-2 gene expression in human lung epithelial cancer cells: coupled mRNA stabilization and posttranscriptional inhibition. Biol Pharm Bull 33: 1170-1175, 2010.

16. Qu C, Liang Z, Huang J, et al: miR-205 determines the radioresistance of human nasopharyngeal carcinoma by directly targeting PTEN. Cell Cycle 11: 785-796, 2012.

17. Pan Y, Zhang Q, Tian L, et al: Jab1/CSN5 negatively regulates p27 and plays a role in the pathogenesis of nasopharyngeal carcinoma. Cancer Res 72: 1890-1900, 2012.

18. Pan Y, Zhang Q, Atsaves V, Yang H and Claret FX: Suppression of Jab1/CSN5 induces radio- and chemo-sensitivity in nasopharyngeal carcinoma through changes to the DNA damage and repair pathways. Oncogene: Jul 16,2012 (Epub ahead of print).

19. Yang $\mathrm{H}$, Wen $Y Y$, Zhao $R$, et al: DNA damage-induced protein 14-3-3 sigma inhibits protein kinase B/Akt activation and suppresses Akt-activated cancer. Cancer Res 66: 3096-3105, 2006.

20. Matsuo K, Gray MJ, Yang DY, et al: The endoplasmic reticulum stress marker, glucose-regulated protein-78 (GRP78) in visceral adipocytes predicts endometrial cancer progression and patient survival. Gynecol Oncol 128: 552-559, 2012.
21. Eizirik DL, Miani M and Cardozo AK: Signalling danger: endoplasmic reticulum stress and the unfolded protein response in pancreatic islet inflammation. Diabetologia 56: 234-241, 2012.

22. Hutzen B, Friedman L, Sobo M, et al: Curcumin analogue GO-Y030 inhibits STAT3 activity and cell growth in breast and pancreatic carcinomas. Int J Oncol 35: 867-872, 2009.

23. Friedman L, Lin L, Ball S, et al: Curcumin analogues exhibit enhanced growth suppressive activity in human pancreatic cancer cells. Anticancer Drugs 20: 444-449, 2009.

24. Naidu MD, Mason JM, Pica RV, Fung H and Pena LA: Radiation resistance in glioma cells determined by DNA damage repair activity of Ape1/Ref-1. J Radiat Res 51: 393-404, 2010.

25. Zou W, Yue P, Khuri FR and Sun SY: Coupling of endoplasmic reticulum stress to CDDO-Me-induced up-regulation of death receptor 5 via a CHOP-dependent mechanism involving JNK activation. Cancer Res 68: 7484-7492, 2008

26. Zhang K and Kaufman RJ: Signaling the unfolded protein response from the endoplasmic reticulum. J Biol Chem 279: 25935-25938, 2004.

27. Liu D, Yin H and Zhang M: Signaling pathways involved in endoplasmic reticulum stress-induced neuronal apoptosis. Int J Neurosci 123: 155-162, 2012.

28. Bobrovnikova-Marjon E, Grigoriadou C, Pytel D, et al: PERK promotes cancer cell proliferation and tumor growth by limiting oxidative DNA damage. Oncogene 29: 3881-3895, 2010.

29. Yamazaki T, Sasaki N, Nishi M and Takeshima H: Facilitation of DNA damage-induced apoptosis by endoplasmic reticulum protein mitsugumin23. Biochem Biophys Res Commun 392: 196-200, 2010. 